\title{
Twin-Twin Transfusion Syndrome Presenting as Polyhydramnios in Both Fetuses Secondary to Spontaneous Microseptostomy
}

\author{
David N. Hackney, MD, MS¹ Nahla Khalek, MD² Julie Moldenhauer, MD² Tulin Ozcan, MD \\ ${ }^{1}$ Department of Obstetrics and Gynecology, University of Rochester \\ School of Medicine, Rochester, New York \\ ${ }^{2}$ The Center for Fetal Diagnosis and Treatment, The Children's \\ Hospital of Philadelphia, Philadelphia, Pennsylvania

\begin{abstract}
Address for correspondence David N. Hackney, MD, MS, Division of Maternal Fetal Medicine, Department of Obstetrics and Gynecology, University Hospitals Case Medical Center, MacDonald Women's Hospital, 11100 Euclid Avenue, Cleveland, OH 44106

(e-mail: David.Hackney@UHhospitals.org).
\end{abstract}

Am J Perinatol Rep 2013;3:83-86.

\begin{abstract}
Keywords

- twin-twin transfusion syndrome

- polyhydramnios

- microseptostomy

- monochorionic

- multifetal gestation

The presence of polyhydramnios and oligohydramnios is pathognomonic for twin-twin transfusion syndrome (TTTS). However, polyhydramnios of both twins can exist in TTTS in the setting of a septostomy of the dividing membrane. In prior reported cases of dual polyhydramnios TTTS, the septostomy was identified through either ultrasound or fetoscopy thus helping to establish the diagnosis of TTTS with an unusual presentation. The presented case is a set of monochorionic, diamniotic twins who presented initially with dual polyhydramnios. Subsequent ultrasound and clinical and pathologic findings were otherwise consistent with TTTS. Unlike prior reported cases, a septostomy of the dividing membrane was never identified with ultrasound or even on post delivery placental examination. However, microseptostomies were demonstrated due to the transfer of indigo carmine between the amniotic sacs at amniocentesis. Thus in the setting of TTTS concern, the diagnosis should be considered with dual polyhydramnios even if a septostomy cannot be identified.
\end{abstract}

A 30-year-old, gravid 4, parity 2012 presented for care in the first trimester with a confirmed monochorionic, diamniotic twin gestation. Biweekly ultrasounds were initiated for twin-twin transfusion syndrome (TTTS) surveillance. Between 16 and 20 weeks, both fetuses demonstrated normal growth and amniotic fluid volumes. Fetal anatomic evaluation did not identify any congenital malformations. At an estimated gestational age of 20 weeks 3 days, polyhydramnios was noted for both fetuses, with the maximum vertical pocket (MVP) being $10.1 \mathrm{~cm}$ for fetus $A$ and $10.3 \mathrm{~cm}$ for fetus $B$. Additionally, the bladder in fetus A could not be visualized, and the bladder of fetus B was subjectively enlarged. Persistent absent end diastolic flow (AEDF) in the umbilical artery was noted on Doppler sonography for fetus B. Both fetuses demonstrated appropriate growth and were concordant for gestational age.

received

November 29, 2012 accepted after revision

February 20, 2013

published online

May 21, 2013
At $21^{5 / 7}$ weeks, the bladder of twin A was still not visualized, polyhydramnios had significantly worsened for both fetuses (MVP 15.0 and $15.4 \mathrm{~cm}$ for fetus A and B, respectively), and persistent reversed end diastolic flow (REDF) in the umbilical artery was noted for fetus B. The patient was referred to a fetal treatment center secondary to the concern for a nonclassic presentation of TTTS. Ultrasound findings were confirmed, and the possibility of TTTS was discussed.

At $22^{3 / 7}$ weeks, the patient underwent amniocentesis and subsequent 1-L amnioreduction with injection of indigo carmine into the amniotic sac of fetus A. Amniocentesis and amnioreduction were then performed for fetus B. Spontaneous communication between the two amniotic sacs was confirmed when dye was noted to be present in the amniotic fluid removed from fetus B (-Fig. 1). Of note, the membranes had previously appeared intact on multiple prior sonographic
Copyright (c) 2013 by Thieme Medical Publishers, Inc., 333 Seventh Avenue, New York, NY 10001, USA. Tel: +1(212) 584-4662.
License terms

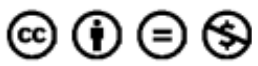

10.1055/s-0033-1343790. ISSN 2157-6998. 


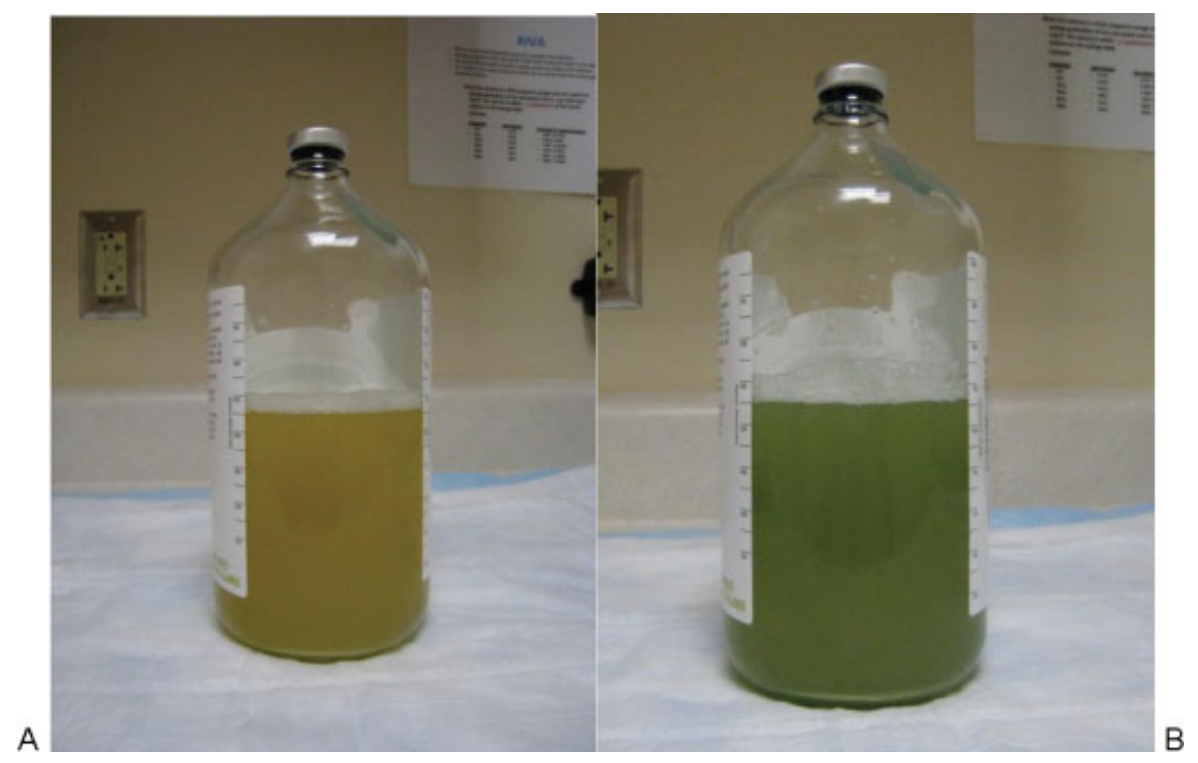

Fig. 1 (A) The amniotic fluid of twin A was initially noted to be clear, though indigo carmine was injected into the sac at the conclusion of the procedure. However, (B) indigo carmine was present in the amniotic fluid of twin B during the amniocentesis for that twin, as evidenced by the color, demonstrating the presence of a microseptostomy despite no gross communication being present either on ultrasound or even pathologic evaluation of the membranes postdelivery.

evaluations, and so the etiology was felt to be a spontaneous, idiopathic microseptostomy.

Follow-up ultrasound evaluation at $22^{5 / 7}$ weeks demonstrated no change in postprocedural fluid volumes and persistent REDF for fetus $B$; however, a small cycling bladder was now seen for fetus $A$. Repeat evaluation at $23^{1 / 7}$ weeks revealed AEDF in the ductus venosus of fetus $B$. The patient was presented with the option of either serial amnioreduction for maternal comfort or selective reduction of fetus B and opted for intermittent amnioreduction and returned to her local provider.

Repeat examination at $23^{5 / 7}$ weeks demonstrated worsening of the TTTS with a recurrence of polyhydramnios (MVP 15.9 and $15.5 \mathrm{~cm}$ for fetus A and B, respectively) and nonvisualization of the bladder of fetus A. Fetus B had persistent REDF and an enlarged heart with decreased contractility. The patient was informed of the guarded prognosis and opted to return to the referral center the next day, where she was subsequently diagnosed with a dual demise and underwent labor induction. The subsequent placental pathology demonstrated a total of four arterial-venous anastomoses, two present in each direction. The fetal membranes appeared visually intact on gross examination. The final karyotype was normal and the fetal autopsy was also consistent with a diagnosis of TTTS with evidence of chronic vascular overload in twin B (plethoric appearance, cardiomegaly, and dilated bladder) and anemia in twin A (pallor, reduced bladder and heart size).

\section{Discussion}

The presence of polyhydramnios and oligohydramnios in a set of monochorionic twins is both pathognomonic for and lies at the heart of the sonographic definition of TTTS. ${ }^{1}$ However,
TTTS can both present in monoamniotic gestations or persist after intentional septostomy. ${ }^{2}$ Thus in the setting of a communication between the amniotic cavities of the two fetuses, the underlying pathophysiology of TTTS can exist and worsen in the absence of a classic polyhydramnios and oligohydramnios appearance.

With a known monoamniotic gestation or after an intentional septostomy, the provider is aware of the communication between the amniotic cavities and thus the potential that the donor may not manifest oligohydramnios. In the presented case, however, the patient had not previously undergone an invasive procedure and a communication could not be identified sonographically. Though uncommon, spontaneous septostomies have been reported in diamniotic twin gestations, ${ }^{3}$ even resulting in cord entanglement at the time of delivery. ${ }^{4}$ Yoshimura et al and Chmait et al previously reported cases of TTTS in which the donor twin manifested either polyhydramnios or normal amniotic fluid volumes secondary to the occurrence of a spontaneous septostomy. ${ }^{5,6}$ In these cases, the septostomy had been apparent either prior to delivery or at the time of fetoscopy. In the presented case, the providers were aware of the potential for the unusual TTTS presentation to have been the result of a spontaneous septostomy, and thus several careful attempts were made by different providers to identify one. No septostomy, however, was ever directly visualized prior to delivery or even on gross examination of the placenta itself. The fact that indigo carmine was able to transfer from the sac of one twin to the other demonstrated the presence of spontaneous microseptostomies that were too small to visualize. The sonographic appearance, unfortunate clinical course, and placental pathologic findings all otherwise supported the diagnosis of TTTS.

In conclusion, practitioners managing patients with monochorionic-diamniotic twin gestations should be aware 
of the potential for nonclassic sonographic manifestations of TTTS secondary to the occurrence of spontaneous microseptostomies. This is true even if one is unable to directly visualize such septostomies sonographically, because small amniotic defects may elude detection, as in the current case. If the appearance of the fetuses is otherwise consistent with TTTS, the presence of an undiagnosed septostomy has the potential to generate diagnostic confusion and potentially delay the onset of appropriate therapy. The presence or absence of microseptostomies can be determined through the use of indigo carmine.

\section{References}

1 Quintero RA, Morales WJ, Allen MH, Bornick PW, Johnson PK, Kruger M. Staging of twin-twin transfusion syndrome. J Perinatol 1999;19(8 Pt 1):550-555
2 Gallot D, Saulnier JP, Savary D, Laurichesse-Delmas H, Lemery D. Ultrasonographic signs of twin-twin transfusion syndrome in a monoamniotic twin pregnancy. Ultrasound Obstet Gynecol 2005;25:308-309

3 Gilbert WM, Davis SE, Kaplan C, Pretorius D, Merritt TA, Benirschke K. Morbidity associated with prenatal disruption of the dividing membrane in twin gestations. Obstet Gynecol 1991;78:623-630

4 Sherer DM, Bitton C, Stimphil R, Dalloul M, Khoury-Collado F, Abulafia O. Cord entanglement of monochorionic diamniotic twins following spontaneous antepartum septostomy sonographically simulating a true knot of the umbilical cord. Ultrasound Obstet Gynecol 2005;26:676-678

5 Yoshimura K, Aiko Y, Inagaki H, Nakata M, Hachisuga T. Prenatal spontaneous disruption of the dividing membrane in monochorionic diamniotic twins detected at the time of fetoscopic laser photocoagulation. J Obstet Gynaecol Res 2009;35:1129-1131

6 Chmait RH, Aghajanian P, Kontopoulos EV, Quintero RA. Prenatal diagnosis of spontaneous septostomy of the dividing membranes in complicated monochorionic diamniotic multiple gestations. J Ultrasound Med 2009;28:663-668 